\title{
Extended Poisson-Fréchet Distribution: Mathematical Properties and Applications to Survival and Repair Times
}

\author{
M. S. Hamed ${ }^{1.2}$ \\ ${ }^{1}$ Management Information System Department, Taibah University, Saudi \\ Arabia. \\ ${ }^{2}$ Department of Statistics, Mathematics and Insurance, Benha University, \\ Egypt
}

\begin{abstract}
In this paper, a new four parameter zero truncated Poisson Fréchet distribution is defined and studied. Various structural mathematical properties of the proposed model including ordinary moments, incomplete moments, generating functions, order statistics, residual and reversed residual life functions are investigated. The maximum likelihood method is used to estimate the model parameters. We assess the performance of the maximum likelihood method by means of a numerical simulation study. The new distribution is applied for modeling two real data sets to illustrate empirically its flexibility.

Keywords: Zero Truncated Poisson Distribution; Fréchet Distribution; Maximum Likelihood Estimation; Simulation; Generating Function; Moments; Order Statistics.
\end{abstract}

\section{Genesis, physical motivation and justification}

Assume that $X_{1}, X_{2}, \ldots, X_{n}$ is a finite sequence of independent and identically distributed random variables (iid rvs) with common cumulative distribution function (CDF). One of the most interesting statistics is the sample maximum

$$
M_{n}=X=\max _{i=1}^{N}\left\{X_{i}\right\}
$$

One is interested in the behavior of $M_{n}$ as the sample size $n$ increases to infinity, then

$$
\begin{aligned}
\operatorname{Pr}\left\{M_{n} \leq x\right\} & =\operatorname{Pr}\left\{X_{1} \leq x, \ldots, X_{n} \leq x,\right\} \\
& =\operatorname{Pr}\left\{X_{1} \leq x\right\} \ldots p_{r}\left\{X_{n} \leq x\right\} \\
& =G(x)^{n} .
\end{aligned}
$$


Suppose there are sequences of constants $\left\{a_{n}>0\right\}$ and $\left\{b_{n}\right\}$ such that

$$
\left.\operatorname{Pr}\left\{\left[\left(M_{n}-b_{n}\right) a_{n}^{-1}\right] \leq x\right\} \rightarrow G(x)\right|_{(n \rightarrow \infty)} .
$$

Then, if $G(x)$ is a non-degenerate CDF, then it will belong to one of the three following fundamental types of classic extreme value family:

1-Gumbel (Gum) model (Type I extreme value distribution);

2-Fréchet (Fr) model (Type II extreme value distribution);

3-Weibull (W) model (Type III extreme value distribution).

The extreme value theory focuses on the behavior of the block maxima or minima. The extreme value theory was firstly introduced by Fréchet (1927) then followed by Von Mises (1936), Gnedenko (1943), Von Mises (1964), Kotz and Johnson (1992), among others. The Fr distribution is one of the important distributions in extreme value theory and has many applications such as accelerated life testing, earthquakes, floods, horse racing, rainfall, queues in supermarkets, wind speeds and sea waves. For more details about the Fr distribution and its applications, see Kotz and Nadarajah (2000). Moreover, applications of this distribution in various fields are given in Harlow (2002). Recently, some extensions of the Fr distribution were considered. The exponentiated Fr by Nadarajah and Kotz (2003), beta Fr by Nadarajah and Gupta (2004), Nadarajah and Kotz (2008) and Zaharim et al. (2009), beta Fr (Barreto-Souza et al. (2011) and Mubarak (2013)), Marshall-Olkin Fr (Krishna et.al. (2013)), transmuted Fr (Mahmoud and Mandouh (2013)), gamma extended Fr (da Silva et al. (2013)), transmuted exponentiated Fr (Elbatal et al. (2014)), transmuted Marshall-Olkin Fr (Afify et al. (2015)), transmuted exponentiated generalized Fr (Yousof et al. (2015)), beta exponential Fr (Mead et al. (2016)), Weibull Fr (Afify et al. (2016b)), Kumaraswamy Marshall-Olkin Fr (Afify et al. (2016b)), Kumaraswamy transmuted Marshall-Olkin Fr (Yousof et al. (2016)), beta transmuted Fr by Afify et al. (2016c), odd Lindley Fréchet distribution the (Korkmaz et al. (2017)), Transmuted Topp-Leone Fr (Yousof et al. (2017)), Topp Leone Generated Fr (Yousof et al. (2018b)) and Odd log-logistic Féchet (Yousof et al. (2018a)), among others.

The goal of this paper is to propose a new generalization of the Topp Leone Fr (TL-Fr) distribution (Yousof et al. (2018b)) using the zero-truncated Poisson (ZTP) model. the probability density function (PDF) and CDF of TL-Fr distribution are given by

$$
\begin{aligned}
g(x)= & 2 \theta \beta \delta^{\beta} x^{-(\beta+1)} \exp \left[-\left(\delta x^{-1}\right)^{\beta}\right] \exp \left[-(\theta-1)\left(\delta x^{-1}\right)^{\beta}\right] \\
& \times\left\{1-\exp \left[-\left(\delta x^{-1}\right)^{\beta}\right]\right\}\left\{2-\exp \left[-\left(\delta x^{-1}\right)^{\beta}\right]\right\}^{\theta-1}, 1
\end{aligned}
$$

and

$$
G(x)=\left(\exp \left[-\left(\delta x^{-1}\right)^{\beta}\right]\left\{2-\exp \left[-\left(\delta x^{-1}\right)^{\beta}\right]\right\}\right)^{\theta},
$$

respectively, where $\delta>0$ is a scale parameter and $\beta, \theta>0$ is a shape parameter. The ZTP distribution is a discrete probability model whose support is the set of only the positive integers $\left(\mathbf{I}^{(+)}\right)$with probability mass function $(\mathrm{PMF})$ of $N$ given by

$$
P\left(N=\left.n\right|_{n \in \mathbf{I}^{(+)}}\right)=\frac{\exp (-\alpha) \alpha^{n}}{\Delta_{(\alpha)} \Gamma(1+n)} .
$$


Suppose that a system has $N$ subsystems functioning independently at a given time where $N$ follows the ZTP distribution with parameter $\alpha$. The expected value $(\mathbf{E}(N \mid \alpha))$ and variance $(\mathbf{V}(N \mid \alpha))$ are, respectively, given by

$$
\mathbf{E}\left(\left.N\right|_{\alpha, n \in \mathbf{I}^{(+)}}\right)=\alpha \Delta_{(\alpha)}^{-1},
$$

where $\Delta_{(\alpha)}=1-\exp (-\alpha)$ and

$$
\mathbf{V}\left(\left.N\right|_{\alpha, n \in \mathbf{I}^{(+)}}\right)=\alpha(1+\alpha) \Delta_{(\alpha)}^{-1}-\alpha^{2} \Delta_{(\alpha)}^{-2}=\mathbf{E}(N \mid \alpha)[1+\alpha-\mathbf{E}(N \mid \alpha)] .
$$

The ZTP is known also as the positive Poisson distribution or the conditional Poisson distribution. It is the conditional probability distribution of a Poisson distributed rv, given that the value of the rv is not zero. Thus it is impossible for a ZTP rv to be zero.

Suppose that the failure time of each subsystem has the TL-Fr model defned by PDF and CDF in (1) and (2). Let $Y_{i}$ denote the failure time of the $i^{(t h)}$ subsystem and let

$$
X=\min _{i=1}^{N}\left\{Y_{i}\right\} \text { or } X=\max _{i=1}^{N}\left\{Y_{i}\right\}
$$

then the conditional CDF of $X$ given $N$ can be written as

$$
F(x \mid N)=1-\operatorname{Pr}(X>x \mid N)=1-\operatorname{Pr}\left(Y_{1}>x\right)^{N}=1-\left(1-G_{T L-F r}^{(\theta, \beta, \delta)}(x)\right)^{N},
$$

therefore, the marginal CDF of $X$ can be expressed as

$$
\left.F(x)\right|_{(\alpha \in \mathbf{R})}=\frac{1-\exp \left\{-\alpha \exp \left[-\theta\left(\delta x^{-1}\right)^{\beta}\right]\left\{2-\exp \left[-\left(\delta x^{-1}\right)^{\beta}\right]\right\}^{\theta}\right\}}{\Delta_{(\alpha)}}
$$

equation (5) is called the CDF of the zero truncated Poisson Topp Leone Fr (ZTPTL-Fr) model. The corresponding PDF of (5) reduces to

$$
\begin{aligned}
\left.f(x)\right|_{(\alpha \in \mathbf{R})}= & \frac{2 \alpha \theta \beta \delta^{\beta}}{\Delta_{(\alpha)} x^{\beta+1}} \exp \left[-\theta\left(\delta x^{-1}\right)^{\beta}\right] \\
& \times\left\{1-\exp \left[-\left(\delta x^{-1}\right)^{\beta}\right]\right\}\left\{2-\exp \left[-\left(\delta x^{-1}\right)^{\beta}\right]\right\}^{\theta-1} \\
& \times \underbrace{\exp \left(-\alpha \exp \left[-\theta\left(\delta x^{-1}\right)^{\beta}\right]\left\{2-\exp \left[-\left(\delta x^{-1}\right)^{\beta}\right]\right\}^{\theta}\right)}_{A(x)}, 6
\end{aligned}
$$

Then we provide a linear mixture for the ZTPTL-Fr density function in (6). Expanding the quantity $A(x)$ in power series, we can write

$$
A(x)={ }_{\tau=0}^{\infty} \frac{(-1)^{\tau} \alpha^{\tau}\left\{2-\exp \left[-\left(\delta x^{-1}\right)^{\beta}\right]\right\}^{\theta \tau}}{\tau ! \exp \left[\theta \tau\left(\delta x^{-1}\right)^{\beta}\right]}
$$


then

$$
\begin{aligned}
f(x)= & \underset{\tau=0}{\infty} \frac{(-1)^{\tau} \alpha^{\tau+1} \theta 2^{(1+\tau) \theta} \beta \delta^{\beta}}{\tau !\left(1-\Delta_{(\alpha)}\right) x^{\beta+1}} \exp \left[-[(1+\tau) \theta]\left(\delta x^{-1}\right)^{\beta}\right] \\
& \times\left\{1-\exp \left[-\left(\delta x^{-1}\right)^{\beta}\right]\right\}\left\{1-2^{-1} \exp \left[-\left(\delta x^{-1}\right)^{\beta}\right]\right\}, 7
\end{aligned}
$$

consider the power series

$$
\left(1-\frac{a_{1}}{a_{2}}\right)^{a_{3}}=\underset{m=0}{\infty} \frac{\Gamma\left(1+a_{3}\right)}{m ! \Gamma\left(1+a_{3}-m\right)}\left(-\frac{a_{1}}{a_{2}}\right)^{m},
$$

which holds for $\left|\frac{a_{1}}{a_{2}}\right|<1$ and $q>0$ real non-integer. Using the power series in (8) and after some algebra the PDF of the ZTPTL-Fr in (7) can be expressed as

$$
f(x)=\underset{\tau, \kappa=0}{\infty}\left[\begin{array}{cc}
\mathbf{c}_{\tau, \kappa} & \pi_{(1+\tau) \theta+\kappa}(x ; \beta, \delta) \\
-\mathbf{c}_{\tau, \kappa}^{\star} & \pi_{1+\kappa+(1+\tau) \theta}(x ; \beta, \delta)
\end{array}\right],
$$

where

$$
\begin{aligned}
\mathbf{c}_{\tau, \kappa} & =\frac{\theta \alpha^{\tau+1}(-1)^{\tau+\kappa} 2^{(1+\tau) \theta-\kappa}}{\tau ! \Delta_{(\alpha)}[(1+\tau) \theta+\kappa]}\left(\begin{array}{c}
-1+(1+\tau) \theta \\
\kappa
\end{array}\right), \\
\mathbf{c}_{\tau, \kappa}^{\star} & =\frac{\theta \alpha^{\tau+1}(-1)^{\tau+\kappa} 2^{(1+\tau) \theta-\kappa}}{\tau ! \Delta_{(\alpha)}[1+\kappa+(1+\tau) \theta]}\left(\begin{array}{c}
-1+(1+\tau) \theta \\
\kappa
\end{array}\right),
\end{aligned}
$$

the function $\pi_{(1+\tau) \theta+\kappa}(x ; \beta, \delta)$ is the Fr density with scale parameter $\delta[(1+\tau) \theta+\kappa]^{\frac{1}{\beta}}$ and shape parameter $\beta$ and $\pi_{1+\kappa+(1+\tau) \theta}(x ; \beta, \delta)$ is the Fr density with scale parameter $\delta[1+\kappa+(1+\tau) \theta]^{\frac{1}{\beta}}$ and shape parameter $\beta$. Equation (9) reveals that the density of $X$ can be expressed as a double linear mixture of Fr densities. So, several of its structural properties can be obtained from Equation (9) and those properties of the Fr distribution. By integrating (9), we obtain the same mixture representation

$$
F(x)=\underset{\tau, \kappa=0}{\infty}\left[\begin{array}{cc}
\mathbf{c}_{\tau, \kappa} & \Pi_{(1+\tau) \theta+\kappa}(x ; \beta, \delta) \\
-\mathbf{c}_{\tau, \kappa}^{\star} & \Pi_{1+\kappa+(1+\tau) \theta}(x ; \beta, \delta)
\end{array}\right],
$$

where $\Pi_{(1+\tau) \theta+\kappa}(x ; \beta, \delta)$ is the CDF of the Fr model with scale parameter $\delta[(1+\tau) \theta+\kappa]^{\frac{1}{\beta}}$ and shape parameter $\beta$ and $\Pi_{1+\kappa+(1+\tau) \theta}(x ; \beta, \delta)$ is the CDF of the Fr model with scale parameter $\delta[1+\kappa+(1+\tau) \theta]^{\frac{1}{\beta}}$ and shape parameter $\beta$. The hazard rate function (HRF) can be derived as $f(x) /[1-F(x)]$. Figure 1 gives some plots of the ZTPTL-Fr PDF and HRF for some parameter values.

The justification for the practicality of the ZTPTL-Fr lifetime model is based on the wider use of the Fr model. As well as we are motivated to introduce the ZTPTL-Fr lifetime model because it exhibits a unimodal hazard rate as illustrated in Figure 1(b). It is shown in above that the ZTPTL-Fr lifetime model can be viewed as a double linear mixture of the Fr distributions. It can be viewed as a suitable model for fitting the unimodal and right skewed data. The ZTPTL-Fr model provide adequate fits as compared to other Fr models in both applications with small values for AIC and BIC. The proposed ZTPTL-Fr model is much better than the Kumaraswamy-Marshall-Olkin Fr, Marshall-Olkin Kumaraswamy Fr, Marshall-Olkin Fr, Kumaraswamy Fr, beta Fr, Marshall-Olkin inverse exponential, Marshall-Olkin inverse Rayleigh, 

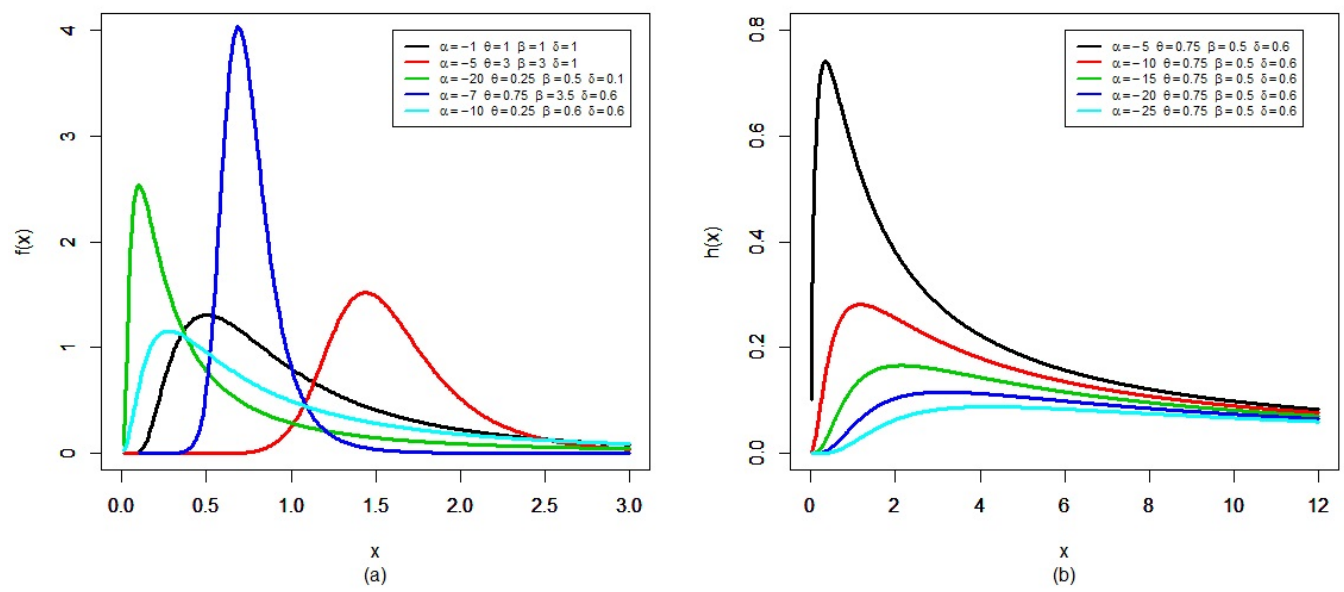

Figure 1: Plots of the ZTPTL-Fr PDF and HRF for some parameter values.

exponentiated Fr and Fr models, so the ZTPTL-Fr model is a suitable alternative to these models for modeling survival times data as illustrated in application 1. As well as the proposed ZTPTL-Fr lifetime model is much better than the Topp Leone Generated Fr, Fr, Kumaraswamy Fr, exponentiated Fr, beta Fr, Transmuted Fr, Marshall-Olkin Fr and Mcdonald Fr models, so the ZTPTL-Fr model is a suitable alternative to these models for modeling repair times data as illustrated in application 2.

The rest of the paper is outlined as follows. In Section 2, we derive some statistical properties for the new model. Maximum likelihood estimation of the model parameters is addressed in Section 3. Simulation results are presented in Section 4. Two applications to real data sets to illustrate the importance of the new family are provided in Section 5. Finally, we offer some concluding remarks in Section 6.

\section{Mathematical properties}

\subsection{Moments and incomplete moments}

The $r^{(t h)}$ ordinary moment of $X$ is given by

$$
\mu_{r}^{\prime}=\mathbf{E}\left(X^{r}\right)=\int_{-\infty}^{\infty} x^{r} f(x) d x
$$

then we obtain

$$
\mu_{r}^{\prime}=\left.\delta^{r} \Gamma\left(1-\frac{r}{\beta}\right) \sum_{\tau, \kappa=0}^{\infty}\left[\mathbf{c}_{\tau, \kappa}^{(1)}-\mathbf{c}_{\tau, \kappa}^{(2)}\right]\right|_{(\beta>r)},
$$


where

$$
\mathbf{c}_{\tau, \kappa}^{(1)}=\frac{\mathbf{c}_{\tau, \kappa}}{[(1+\tau) \theta+\kappa]^{-\frac{r}{\beta}}},
$$

and

$$
\mathbf{c}_{\tau, \kappa}^{(2)}=\frac{\mathbf{c}_{\tau, \kappa}^{\star}}{[1+\kappa+(1+\tau) \theta]^{-\frac{r}{\beta}}} .
$$

The constants $c_{\tau, \kappa}$ and $c_{\tau, \kappa}^{\star}$ have been defined before in Section 1, and

$$
\left.\Gamma(1+\varphi)\right|_{\left(\varphi \in \mathbb{R}^{+}\right)}=\int_{0}^{\infty} x^{\varphi} \exp (-t) d x=\varphi !=_{w=0}^{\varphi-1}(\varphi-w) .
$$

Setting $r=1$ in (11), we have the mean of $X\left(\mu^{\prime}\right)$. The last integration can be computed numerically for most parent distributions. The skewness and kurtosis measures can be calculated from the ordinary moments using well-known relationships. The $r^{(t h)}$ incomplete moment, say $\Upsilon_{r}(t)$, of $X$ can be expressed from (9) as

$$
\Upsilon_{r}(t)=\int_{-\infty}^{t} x^{r} f(x) d x
$$

then

$$
\Upsilon_{r}(t)=\left.\delta^{r} \sum_{\tau, \kappa=0}^{\infty}\left[\begin{array}{c}
\mathbf{c}_{\tau, \kappa}^{(1)} \gamma\left(1-\frac{r}{\beta},[(1+\tau) \theta+\kappa]\left(\frac{\delta}{t}\right)^{\beta}\right) \\
-\mathbf{c}_{\tau, \kappa}^{(2)} \gamma\left(1-\frac{r}{\beta},[1+\kappa+(1+\tau) \theta]\left(\frac{\delta}{t}\right)^{\beta}\right)
\end{array}\right]\right|_{(\beta>r)},
$$

where

$$
\begin{aligned}
\gamma(\zeta, z) & =\int_{0}^{z} t^{\zeta-1} \exp (-t) d t \\
& =\frac{z^{\zeta}}{\zeta}\left\{{ }_{1} \mathbf{F}_{1}[\zeta ; \zeta+1 ;-z]\right\} \\
& =\left.\sum_{\nu=0}^{\infty} \frac{(-1)^{\nu}}{\nu !(\zeta+\nu)} z^{\zeta+\nu}\right|_{(\zeta \neq 0,-1,-2, \ldots)},
\end{aligned}
$$

where ${ }_{1} \mathbf{F}_{1}[\cdot]$ is a confluent hypergeometric function, which can be evaluated by statistical software like $\mathrm{R}$ software.

\subsection{Numerical analysis for the $\mu^{\prime}$, variance $(\mathrm{V}(X))$, skewness $(\operatorname{Sk}(X))$ and kurtosis}

\section{$(\mathrm{Ku}(X))$ measures}

Numerical analysis for the $\mu^{\prime}, \mathbf{V}(X), \mathbf{S k}(X)$ and $\mathbf{K u}(X)$ are listed in Tables 1 and 2 for the ZTPTL-Fr model and for the Fr model respectively for some selected values of parameter $\alpha, \theta, \beta$ and $\delta$ using the $\mathrm{R}$ software. Based on Table 1 we note that:

1- The $\mathbf{S k}(X)$ of the ZTPTL-Fr model is always positive.

2- The $\mathbf{K u}(X)$ of the ZTPTL-Fr model can be more than 3 or less than 3 .

Based on Tables 1 and 2 we note that: The skewness of the ZTPTL-Fr distribution can range in the interval $(1.704,99.032)$, whereas the skewness of the Fr distribution varies only 
in the interval $(1.001,3.53)$. Further, the spread for the ZTPTL-Fr kurtosis is ranging from 0.625235 to 148485.5 , whereas the spread for the Fr kurtosis only varies from 1.002 to 98.8 with the above parameter values.

Table 1: $\mathbf{E}(X), \mathbf{V}(X), \mathbf{S k}(X)$ and $\mathbf{K u}(X)$ of the ZTPTL-Fr model.

\begin{tabular}{|c|c|c|c|c|c|c|c|}
\hline$\delta$ & $\bar{\beta}$ & $\theta$ & $\alpha$ & $\mathbf{E}(X)$ & $\mathbf{V}(X)$ & Sk $(X)$ & $\mathbf{K u}(X)$ \\
\hline 1 & 1.5 & 1.5 & -3 & 2.571567 & 0.9743642 & 99.03197 & 148485.5 \\
\hline 5 & & & & 12.85782 & 24.21726 & 75.966 & 29355.88 \\
\hline 20 & & & & 51.43077 & 379.0067 & 57.35025 & 7170.361 \\
\hline 50 & & & & 128.5695 & 2264.166 & 46.6469 & 2813.691 \\
\hline 200 & & & & 513.7455 & 28255.84 & 37.25156 & 745.9437 \\
\hline 500 & & & & 1276.935 & 89460.14 & 56.69273 & 511.4524 \\
\hline \multirow[t]{5}{*}{100} & 0.5 & 1.75 & -10 & 2375.807 & 4580343 & 1.541674 & 4.744495 \\
\hline & 1 & & & 738.8926 & 453222.2 & 5.88400 & 48.87404 \\
\hline & 1.25 & & & 496.6668 & 119304 & 9.412066 & 130.6124 \\
\hline & 1.5 & & & 381.6909 & 32180.45 & 15.22058 & 310.0421 \\
\hline & 1.75 & & & 318.3019 & 7494.697 & 37.44547 & 995.5315 \\
\hline \multirow[t]{8}{*}{10} & 0.5 & 1 & 5 & 6.333456 & 3333.628 & 86.60225 & 10002.62 \\
\hline & & 5 & & 37.02739 & 17198.3 & 37.36708 & 1887.333 \\
\hline & & 10 & & 74.76474 & 35530.41 & 25.50535 & 889.1923 \\
\hline & & 50 & & 358.0939 & 210585.3 & 9.633289 & 131.465 \\
\hline & & 100 & & 689.3368 & 473059.3 & 6.105031 & 53.45829 \\
\hline & & 200 & & 1303.943 & 1057407 & 3.793141 & 21.14257 \\
\hline & & 500 & & 2820.337 & 2758617 & 1.717923 & 5.869974 \\
\hline & & 1000 & & 4204.399 & 5754855 & 0.1825467 & 0.625235 \\
\hline \multirow[t]{5}{*}{5} & 0.25 & 10 & -10 & 932.3464 & 4975511 & 2.469925 & 8.055438 \\
\hline & & & -5 & 1548.428 & 6149727 & 1.703678 & 4.898315 \\
\hline & & & 5 & 272.3675 & 525808.9 & 7.220045 & 67.53774 \\
\hline & & & 20 & 31.23634 & 469.4178 & 21.24751 & 2997.731 \\
\hline & & & 30 & 21.80845 & 63.51442 & 18.39078 & 327.8126 \\
\hline
\end{tabular}


Table 2: $\mathbf{E}(X), \mathbf{V}(X), \mathbf{S k}(X)$ and $\mathbf{K u}(X)$ of the Fr model.

\begin{tabular}{cc|cccc}
\hline \hline$\delta$ & $\beta$ & $\mathbf{E}(X)$ & $\mathbf{V}(X)$ & $\mathbf{S k}(X)$ & $\mathbf{K u}(X)$ \\
\hline \hline 0.5 & 5 & 0.5821149 & 0.0334404 & 3.535071 & 48.0915 \\
& 10 & 0.5343144 & 0.0055656 & 1.910339 & 10.9774 \\
& 25 & 0.5123659 & 0.0007351 & 1.400443 & 6.85310 \\
& 50 & 0.5059737 & 0.0001736 & 1.264099 & 6.04447 \\
& & & & & \\
2.5 & 5 & 2.910574 & 0.8360089 & 3.535072 & 48.0915 \\
& 10 & 2.671572 & 0.1391401 & 1.910339 & 10.97857 \\
& 50 & 2.529868 & 0.0043398 & 1.264099 & 6.045233 \\
& 75 & 2.519686 & 0.0018938 & 1.221761 & 5.760403 \\
& & & & & \\
4.5 & 5 & 5.239034 & 2.70867 & 3.53507 & 48.0915 \\
5 & 4.5 & 5.950756 & 4.60640 & 4.23885 & 98.8016 \\
10 & 7.5 & 10.97054 & 4.47131 & 2.29491 & 15.5896 \\
50 & 50 & $1.392 \times \mathrm{e}^{-6}$ & 2561.83 & 1.00104 & 1.0028 \\
60 & 20 & 61.8872 & 17.03792 & 1.473884 & 7.33349 \\
60 & 50 & 60.71684 & 2.499703 & 1.2641 & 6.04521 \\
& & & & & \\
\hline \hline
\end{tabular}

\subsection{Moment generating function}

The moment generating function $(\mathrm{MGF}) M_{X}(t)=\mathbf{E}(\exp (t X))$ of $X$ can be derived from equation (9) as

$$
M_{X}(t)=\left.\delta^{r} \Gamma\left(1-\frac{r}{\beta}\right) \sum_{\tau, \kappa, r=0}^{\infty}\left[m_{\tau, \kappa, r}^{(1)}-m_{\tau, \kappa, r}^{(2)}\right]\right|_{(\beta>r)},
$$

where

$$
m_{\tau, \kappa, r}^{(1)}=\frac{t^{r}}{r !} \mathbf{c}_{\tau, \kappa}[(1+\tau) \theta+\kappa]^{\frac{r}{\beta}}
$$

and

$$
m_{\tau, \kappa, r}^{(2)}=\frac{t^{r}}{r !} \mathbf{c}_{\tau, \kappa}^{\star}[1+\kappa+(1+\tau) \theta]^{\frac{r}{\beta}} .
$$

Using the Wright generalized hypergeometric (WGH) function which defined as

$$
{ }_{(p)} \boldsymbol{\Psi}_{(q)}\left[\begin{array}{c}
\left(\delta_{1}, A_{1}\right), \ldots,\left(\delta_{p}, A_{p}\right) \\
\left(\beta_{1}, B_{1}\right), \ldots,\left(\beta_{q}, B_{q}\right)
\end{array} ; x\right]=\sum_{n=0}^{\infty} \frac{\prod_{\kappa=1}^{p} \Gamma\left(\delta_{\kappa}+A_{\kappa} n\right)}{\prod_{\kappa=1}^{q} \Gamma\left(\beta_{\kappa}+B_{\kappa} n\right)} \frac{x^{n}}{n !},
$$

Then, we can write $M(t ; \delta, \beta)$ as

$$
M(t ; \delta, \beta)={ }_{(1)} \boldsymbol{\Psi}_{(0)}\left[\begin{array}{c}
\left(1,-\frac{1}{\beta}\right) \\
-
\end{array} ; \delta t .\right.
$$


Combining (9) and the last equation, we obtain the MGF of $X$ in terms of WGH function, say $M(t)$, as

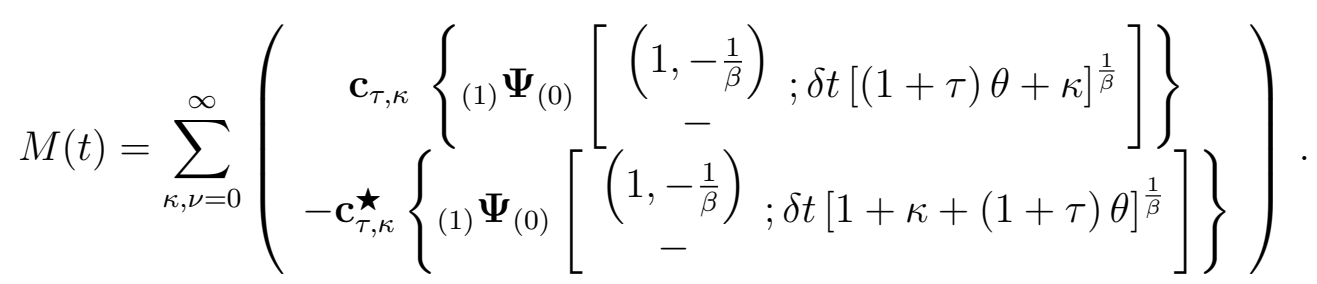

\subsection{Probability weighted moments}

The $(s, r)^{t h}$ PWM of $X$ following the ZTPTL-Fr is formally defined by

$$
\rho_{r, s}=\mathbf{E}\left\{X^{s} F(X)^{r}\right\}=\int_{-\infty}^{\infty} x^{s} F(x)^{r} f(x) d x .
$$

Using equations (5) and (6), we can write

$$
f(x) F(x)^{r}=\sum_{\tau, \kappa=0}^{\infty}\left[\begin{array}{c}
d_{\tau, \kappa} \pi_{(1+\tau) \theta+\kappa}(x ; \beta, \delta) \\
-d_{\tau, \kappa}^{\star} \pi_{1+\kappa+(1+\tau) \theta}(x ; \beta, \delta)
\end{array}\right],
$$

where

$$
d_{\tau, \kappa}=\sum_{\nu=0}^{\infty} \frac{2^{(1+\tau) \theta-\kappa} \theta \alpha^{\tau+1}(-1)^{\tau+\kappa+\nu}(\nu+1)^{\tau}}{\tau ! \Delta_{(\alpha)}^{r+1}[(1+\tau) \theta+\kappa]}\left(\begin{array}{l}
r \\
\nu
\end{array}\right)\left(\begin{array}{c}
-1+(1+\tau) \theta \\
\kappa
\end{array}\right)
$$

and

$$
d_{\tau, \kappa}^{\star}=\sum_{\nu=0}^{\infty} \frac{2^{(1+\tau) \theta-\kappa} \theta \alpha^{\tau+1}(-1)^{\tau+\kappa+\nu}(\nu+1)^{\tau}}{\tau ! \Delta_{(\alpha)}^{r+1}[1+\kappa+(1+\tau) \theta]}\left(\begin{array}{l}
r \\
\nu
\end{array}\right)\left(\begin{array}{c}
-1+(1+\tau) \theta \\
\kappa
\end{array}\right),
$$

then, the $(s, r)^{t h}$ PWM of $X$ can be expressed as

$$
\rho_{r, s}=\left.\delta^{s} \Gamma\left(1-\frac{s}{\beta}\right) \sum_{\tau, \kappa=0}^{\infty}\left[d_{\tau, \kappa}^{(1)}-d_{\tau, \kappa}^{(2)}\right]\right|_{(\beta>s)},
$$

where

$$
d_{\tau, \kappa}^{(1)}=\frac{d_{\tau, \kappa}}{[(1+\tau) \theta+\kappa]^{-\frac{s}{\beta}}}
$$

and

$$
d_{\tau, \kappa}^{(2)}=\frac{d_{\tau, \kappa}^{\star}}{[1+\kappa+(1+\tau) \theta]^{-\frac{s}{\beta}}} .
$$

\subsection{Residual life and reversed residual life functions}

The $n^{(t h)}$ moment of the residual life, say

$$
m_{n}(t)=\mathbf{E}\left[\left.(X-t)^{n}\right|_{(X>t)} ^{(n=1,2, \ldots)}\right],
$$

uniquely determine $F(x)$. The $n^{(t h)}$ moment of the residual life of $X$ is given by

$$
m_{n}(t)=[1-F(t)]^{-1} \int_{t}^{\infty}(x-t)^{n} d F(x),
$$


then

$$
m_{n}(t)=\left.\frac{\delta^{n}}{1-F(t)} \sum_{r=0}^{n} \sum_{\tau, \kappa=0}^{\infty}\left[\begin{array}{c}
b_{\tau, \kappa}^{(1)} \Gamma\left(1-\frac{n}{\beta},[(1+\tau) \theta+\kappa]\left(\frac{\delta}{t}\right)^{\beta}\right) \\
-b_{\tau, \kappa}^{(2)} \Gamma\left(1-\frac{n}{\beta},[1+\kappa+(1+\tau) \theta]\left(\frac{\delta}{t}\right)^{\beta}\right)
\end{array}\right]\right|_{(\beta>n)},
$$

where

$$
\begin{aligned}
&\left.\Gamma(\zeta, z)\right|_{(z>0)}=\int_{0}^{z} t^{\zeta-1} \exp (-t) d t \\
& \sim \frac{z^{\zeta-1}}{\exp (z)}\left[\begin{array}{c}
1 \\
+\frac{\zeta-1}{z} \\
+\frac{(\zeta-1)(\zeta-2)}{z^{2}}+\ldots
\end{array}\right], \\
& \Gamma(\zeta, z)=\Gamma(\zeta)-\gamma(\zeta, z), \\
& b_{\tau, \kappa}^{(1)}=\frac{\mathbf{c}_{\tau, \kappa}}{[(1+\tau) \theta+\kappa]^{\frac{-n}{\beta}}}\left(\begin{array}{c}
n \\
r
\end{array}\right)(-t)^{n-r}
\end{aligned}
$$

and

$$
b_{\tau, \kappa}^{(2)}=\frac{\mathbf{c}_{\tau, \kappa}^{\star}}{[1+\kappa+(1+\tau) \theta]^{\frac{-n}{\beta}}}\left(\begin{array}{l}
n \\
r
\end{array}\right)(-t)^{n-r} .
$$

The $n^{(t h)}$ moment of the reversed residual life, say

$$
M_{n}(t)=\mathbf{E}\left[\left.(t-X)^{n}\right|_{(X \leq t)} ^{(n=1,2, \ldots)} \forall(t>0)\right],
$$

or

$$
M_{n}(t)=F(t)^{-1} \int_{0}^{t}(t-x)^{n} d F(x) .
$$

Then, the $n^{(t h)}$ moment of the reversed residual life of $X$ becomes

$$
M_{n}(t)=\left.\delta^{n} F(t)^{-1} \sum_{r=0}^{n} \sum_{\tau, \kappa=0}^{\infty}\left[\begin{array}{c}
\xi_{\tau, \kappa}^{(1)} \gamma\left(1-\frac{n}{\beta},[(1+\tau) \theta+\kappa]\left(\frac{\delta}{t}\right)^{\beta}\right) \\
-\xi_{\tau, \kappa}^{(2)} \gamma\left(1-\frac{n}{\beta},[1+\kappa+(1+\tau) \theta]\left(\frac{\delta}{t}\right)^{\beta}\right)
\end{array}\right]\right|_{(\beta>n)},
$$

where

$$
\xi_{\tau, \kappa}^{(1)}=\frac{\mathbf{c}_{\tau, \kappa}}{[(1+\tau) \theta+\kappa]^{\frac{-n}{\beta}}}(-1)^{r} t^{n-r},
$$

and

$$
\xi_{\tau, \kappa}^{(2)}=\frac{\mathbf{c}_{\tau, \kappa}^{\star}}{[1+\kappa+(1+\tau) \theta]^{\frac{-n}{\beta}}}(-1)^{r} t^{n-r} .
$$

The mean waiting time or mean inactivity time (MIT) also called the mean reversed residual life function is given by $M_{1}(t)=\mathbf{E}\left[\left.(t-X)\right|_{(X \leq t)}\right]$, and it represents the waiting time elapsed since the failure of an item on condition that this failure had occurred in $(0, t)$. The MIT of the ZTPTL-Fr distribution can be obtained easily by setting $n=1$ in the above equation. 


\subsection{Order statistics and quantile spread ordering}

Let $X_{1}, \ldots, X_{n}$ be a random sample (RS) from the ZTPTL-Fr distribution and let $X_{1: n}, \ldots, X_{n: n}$ be the corresponding order statistics. The PDF of $i^{(t h)}$ order statistic, say $X_{i: n}$, can be written as

$$
f_{i: n}(x)=\frac{f(x)}{\mathrm{B}(i, n-i+1)} \sum_{\kappa=0}^{n-i}(-1)^{\kappa}\left(\begin{array}{c}
n-i \\
\kappa
\end{array}\right) F^{\kappa+i-1}(x),
$$

where $B(\cdot, \cdot)$ is the beta function. Substituting (5) and (6) in equation (13) and using a power series expansion,we get

$$
f(x) F(x)^{\kappa+i-1}=\sum_{w, m=0}^{\infty}\left[\begin{array}{cc}
t_{w, m} & \pi_{(1+w) \theta+m}(x ; \beta, \delta) \\
-t_{w, m}^{\star} & \pi_{1+m+(1+w) \theta}(x ; \beta, \delta)
\end{array}\right],
$$

where

$$
\begin{aligned}
t_{w, m}= & \sum_{\nu=0}^{\infty} \frac{2^{\theta(w+1)-m} \theta \alpha^{w+1}(-1)^{w+m+\nu}(\nu+1)^{w}}{w ! \Delta_{(\alpha)}^{\kappa+i}[\theta(w+1)+m]} \\
& \times\left(\begin{array}{c}
-1+\kappa+i \\
\nu
\end{array}\right)\left(\begin{array}{c}
-1+(1+w) \theta \\
m
\end{array}\right),
\end{aligned}
$$

and

$$
\begin{aligned}
t_{w, m}^{\star}= & \sum_{\nu=0}^{\infty} \frac{2^{\theta(w+1)-m} \theta \alpha^{w+1}(-1)^{w+m+\nu}(\nu+1)^{w}}{w ! \Delta_{(\alpha)}^{\kappa+i}[\theta(w+1)+m+1]} \\
& \times\left(\begin{array}{c}
-1+\kappa+i \\
\nu
\end{array}\right)\left(\begin{array}{c}
-1+(1+w) \theta \\
m
\end{array}\right) .
\end{aligned}
$$

The PDF of $X_{i: n}$ can be expressed as

$$
f_{i: n}(x)=\sum_{\kappa=0}^{n-i} \frac{(-1)^{\kappa}\left(\begin{array}{c}
n-i \\
\kappa
\end{array}\right)}{\mathrm{B}(i, n-i+1)} \sum_{w, m=0}^{\infty}\left[t_{w, m} \pi_{(1+w) \theta+m}(x)-t_{w, m}^{\star} \pi_{1+m+(1+w) \theta}(x)\right] .
$$

The $q^{(t h)}$ ordinary moment of $X_{i: n}$ can be expressed as

$$
\mathbf{E}\left(X_{i: n}^{q}\right)=\left.\delta^{q} \Gamma\left(1-\frac{q}{\beta}\right) \sum_{\kappa=0}^{n-i} \sum_{w, m=0}^{\infty} \frac{(-1)^{\kappa}\left(\begin{array}{c}
n-i \\
\kappa
\end{array}\right)}{\mathrm{B}(i, n-i+1)}\left[t_{w, m, h}^{(1)}-t_{w, m, h}^{(2)}\right]\right|_{(\beta>q)},
$$

where

$$
t_{w, m, h}^{(1)}=\frac{t_{w, m, h}}{[(1+w) \theta+m]^{-\frac{q}{\beta}}}
$$

and

$$
t_{w, m, h}^{(2)}=\frac{t_{w, m, h}^{\star}}{[1+m+(1+w) \theta]^{-\frac{q}{\beta}}} .
$$

The quantile spread (QS) of the rv $T \sim \mathrm{ZTPTL}-\mathrm{Fr}(\alpha, \theta, \beta, \delta)$ is given by

$$
\{\mathbf{Q S}\}_{T}(\eta)=\left[F^{-1}(\eta)-F^{-1}(1-\eta)\right] \mid\left(\eta \in\left(\frac{1}{2}, 1\right)\right),
$$


which implies

$$
\{\mathbf{Q S}\}_{T}(\eta)=\left[S^{-1}(1-\eta)\right]-\left[S^{-1}(\eta)\right],
$$

where

$$
F^{-1}(\eta)=S^{-1}(1-\eta) \text { and } S(T)=1-F(T),
$$

is the survival function. The QS of a any probability distribution describes how the probability mass is placed symmetrically about its median and hence it can be used to formalize concepts such as peakedness and tail weight traditionally associated with the kurtosis. So, it allows us to separate concepts of the kurtosis and peakedness for asymmetric models. Let $T_{1}$ and $T_{2}$ be two rvs following the ZTPTL-Fr model with $\{\mathbf{Q S}\}_{T_{1}}$ and $\{\mathbf{Q S}\}_{T_{2}}$, respectively. Then $T_{1}$ is called smaller than $T_{2}$ in quantile spread order, denoted as $T_{1} \leq_{\{\mathbf{Q S}\}} T_{2}$, if

$$
\left.\{\mathbf{Q S}\}_{T_{1}}(\xi)\right|_{\left(\eta \in\left(\frac{1}{2}, 1\right)\right)} \leq\{\mathbf{Q S}\}_{T_{2}}(\eta),
$$

then we have the following results:

1 -The order $\leq_{\{\mathbf{Q S}\}}$ is a location-free, where $T_{1} \leq_{\{\mathbf{Q S}\}} T_{2}$ if

$$
\left(T_{1}+\kappa\right) \leq\left._{\{\mathbf{Q S}\}} T_{2}\right|_{(\kappa \in \mathbb{R})} .
$$

2 -The order $\leq_{\{\mathbf{Q S}\}}$ is dilative, where

$$
T_{1} \leq\left._{\{\mathbf{Q S}\}} \kappa T_{1}\right|_{(\kappa \geq 1)},
$$

and

$$
T_{2} \leq\left._{\{\mathbf{Q S}\}} \kappa T_{2}\right|_{(\kappa \geq 1)} .
$$

3-Let $F_{T_{1}}$ and $F_{T_{2}}$ be symmetric, then $T_{1} \leq_{\{\mathrm{QS}\}} T_{2}$ if, and only if

$$
F_{T_{1}}^{-1}(\eta) \leq\left. F_{T_{2}}^{-1}(\eta)\right|_{\left(\eta \in\left(\frac{1}{2}, 1\right)\right)} .
$$

4 -The order $\leq_{\{\mathbf{Q S}\}}$ implies ordering of the mean absolute deviation around the median, say $\left.\xi\left(T_{i}\right)\right|_{(i=1,2)}$, the we have

$$
\xi\left(T_{i}\right)=\mathbf{E}\left[\left|T_{i}-\operatorname{Median}\left(T_{i}\right)\right|\right],
$$

where

$$
T_{1} \leq_{\{\mathbf{Q S}\}} T_{2} \Rightarrow \xi\left(T_{1}\right) \leq_{\{\mathbf{Q S}\}} \xi\left(T_{2}\right),
$$

finally $T_{1} \leq_{\{\mathbf{Q S}\}} T_{2}$ if, and only if

$$
\left.-T_{1} \leq_{\{\mathbf{Q S}}\right\}-T_{2} .
$$

\section{Estimation}

Let $x_{1}, \ldots, x_{n}$ be a RS from the ZTPTL-Fr distribution with parameters $\alpha, \theta, \beta$ and $\delta$. Let $\Theta=(\alpha, \theta, \beta, \delta)^{\top}$ be the $4 \times 1$ parameter vector. For determining the maximum likelihood 
estimators (MLEs) of $\boldsymbol{\Theta}$, we have the log-likelihood function

$$
\begin{aligned}
\ell= & \ell(\Theta)=n \log 2+n \log \alpha+n \log \theta+n \log \beta+n \beta \log \delta \\
& -n \log \left(\Delta_{(\alpha)}\right)-(\beta+1) \sum_{i=1}^{n} \log x_{i}-\theta \delta^{\beta} \sum_{i=1}^{n} x_{i}^{-\beta} \\
& -\alpha \sum_{i=1}^{n} \exp \left[-\theta\left(\delta x_{i}^{-1}\right)^{\beta}\right]\left\{2-\exp \left[-\left(\delta x_{i}^{-1}\right)^{\beta}\right]\right\}^{\theta} \\
& +\sum_{i=1}^{n} \log \left\{1-\exp \left[-\left(\delta x_{i}^{-1}\right)^{\beta}\right]\right\}+(\theta-1) \sum_{i=1}^{n} \log \left\{2-\exp \left[-\left(\delta x_{i}^{-1}\right)^{\beta}\right]\right\} .
\end{aligned}
$$

The above log-likelihood function can be maximized numerically by using R (optim), SAS (PROC NLMIXED) or Ox program (sub-routine MaxBFGS), among others. The components of the score vector, $\mathbf{U}(\boldsymbol{\Theta})=\frac{\partial \ell}{\partial \Theta}=\left(\frac{\partial \ell}{\partial \alpha}, \frac{\partial \ell}{\partial \theta}, \frac{\partial \ell}{\partial \beta}, \frac{\partial \ell}{\partial \delta}\right)^{\top}$ are availabe if needed. Setting the nonlinear system of equations $\mathbf{U}_{\alpha}=\mathbf{U}_{\theta}=\mathbf{U}_{\beta}=\mathbf{U}_{\delta}=0$ and solving them simultaneously yields the MLE $\widehat{\Theta}=(\widehat{\alpha}, \widehat{\theta}, \widehat{\beta}, \widehat{\delta})^{\top}$. To solve these equations, it is usually more convenient to use nonlinear optimization methods such as the quasi-Newton algorithm to numerically maximize $\ell$. For interval estimation of the parameters, we obtain the $4 \times 4$ observed information matrix $\mathbf{J}(\boldsymbol{\Theta})=\left\{\frac{\partial^{2} \ell}{\partial r \partial s}\right\}$ (for $r, s=\alpha, \theta, \beta, \delta$ ), whose elements can be computed numerically. Under standard regularity conditions when $n \rightarrow \infty$, the distribution of $\widehat{\boldsymbol{\Theta}}$ can be approximated by a multivariate normal $N_{4}\left(0, \mathbf{J}(\widehat{\boldsymbol{\Theta}})^{-1}\right)$ distribution to construct approximate confidence intervals for the parameters. Here, $\mathbf{J}(\widehat{\boldsymbol{\Theta}})$ is the total observed information matrix evaluated at $\widehat{\boldsymbol{\Theta}}$. The method of the resampling bootstrap can be used for correcting the biases of the MLEs of the model parameters. Good interval estimates may also be obtained using the bootstrap percentile method.

\section{Simulation results}

We present some simulation experiments for some different sample sizes in order to assess the accuracy of the MLEs. Simulating rvs from well defined probability distributions has been discussed in the literature of computational statistics, e.g. the inverse transformation method, the rejection and acceptance sampling technique, etc. The ideal technique for simulating from the ZTPTL-Fr distribution is the inversion method, we can simulate rv $X$ by

$$
X=\delta\left(-\ln \left\{1-\left[1-\left(\frac{-\ln \left\{1-\left[U\left(\Delta_{(\alpha)}\right)\right]\right\}}{\alpha}\right)^{\frac{1}{\theta}}\right]^{\frac{1}{2}}\right.\right.
$$

where $U$ is a uniform random number in $(0,1)$. For selected combinations of $\alpha, \theta, \beta$ and $\delta$ we generate samples of sizes $n=50,100,200,300,500$ and 1000 from the ZTPTL-Fr distribution. We repeat the simulations $N=1000$ times, we use two combinations for the parameter values $(\alpha=2.5, \theta=1.5, \beta=1$ and $\delta=2)$ in order to obtain average estimates (AEs) and mean square errors (MSEs) of the parameters. The empirical results obtained via using the well-known $R$ package are given in Table 3. 
Table 3: Empirical AEs and (MSEs) for $\alpha=2.5, \theta=1.5, \beta=1$ and $\delta=2$.

\begin{tabular}{|c|c|c|c|c|}
\hline \multirow[b]{2}{*}{ Sample size $(n)$} & \multicolumn{4}{|c|}{ AEs and its corresponding (MSEs) } \\
\hline & $\widehat{\alpha}$ & $\widehat{\theta}$ & $\widehat{\beta}$ & $\widehat{\delta}$ \\
\hline 50 & $\begin{array}{c}2.61195 \\
(1.76198)\end{array}$ & $\begin{array}{c}1.68512 \\
(0.981681)\end{array}$ & $\begin{array}{c}1.47851 \\
(1.342916)\end{array}$ & $\begin{array}{c}2.61178 \\
(2.98781)\end{array}$ \\
\hline 100 & $\begin{array}{l}2.59812 \\
(1.3225)\end{array}$ & $\begin{array}{c}1.63221 \\
(0.822465)\end{array}$ & $\begin{array}{c}1.31917 \\
(1.15650)\end{array}$ & $\begin{array}{c}2.52245 \\
(1.15671)\end{array}$ \\
\hline 200 & $\begin{array}{c}2.60112 \\
(1.21391)\end{array}$ & $\begin{array}{c}1.57613 \\
(0.587918)\end{array}$ & $\begin{array}{c}1.11875 \\
(0.89794)\end{array}$ & $\begin{array}{c}2.38751 \\
(0.32292)\end{array}$ \\
\hline 300 & $\begin{array}{c}2.51686 \\
(1.00293)\end{array}$ & $\begin{array}{c}1.54572 \\
(0.44371)\end{array}$ & $\begin{array}{c}1.03218 \\
(.1988569)\end{array}$ & $\begin{array}{l}2.31510 \\
(0.0901)\end{array}$ \\
\hline 500 & $\begin{array}{c}2.50224 \\
(0.08571)\end{array}$ & $\begin{array}{c}1.50319 \\
(0.299718)\end{array}$ & $\begin{array}{c}1.00166 \\
(0.09829)\end{array}$ & $\begin{array}{c}2.0691 \\
(0.00972)\end{array}$ \\
\hline 1000 & $\begin{array}{c}2.50021 \\
(0.000163)\end{array}$ & $\begin{array}{c}1.50027 \\
(0.019226)\end{array}$ & $\begin{array}{c}1.00071 \\
(0.011985)\end{array}$ & $\begin{array}{c}2.00651 \\
(0.000567)\end{array}$ \\
\hline
\end{tabular}

We observe that our estimates are pretty stable especially when $n \geq 300$ and as $n$ increases the MSEs and biases decreases. So, the maximum likelihood method works very well to estimate the model parameters.

\section{Data analysis}

In this section we provide applications of the ZTPTL-Fr distribution using two real data sets. In order to compare the distributions, we consider some criteria like Akaike Information Criterion (AIC) and Bayesian Information Criterion (BIC) value is chosen as the best model to fit the data.

The first data set consists of 72 observations of survival times for Guinea pigs injected with different doses of tubercle bacilli: $12,15,43,44,263,297,341,34148,76,76,81,83,84,85,87$, $58,52,53,73,75,59,60,54,4,24,175,22,234,38,38,70,70,72,175,211,32,62,63,65$, $65,67,68,60,32,33,54,55,56,146,233,258,57,58,60,60,61,91,95,96,98,99,109,110$, $121,127,129,131,143,146,258$ and 376.These data were previously studied by Krishna et al. (2013). We compare the proposed ZTPTL-Fr distribution with other related models namely: the Kumaraswamy Marshall-Olkin Fr (KwMO-Fr), MOKw-Fr, Kw-Fr, MO-Fr, beta -Fr (B-Fr), Exponentiated Fr (E-Fr), Marshall-Olkin Invere Exponential (MOIE), Marshall-Olkin invere Rayleigh (MOIR) and Fr distributions. The second data set (repair times data) represents an active repair times (hours) for an airborne communication transceiver, to be self-contained, this data set is reproduced as follows: $0.2,0.5,0.5,0.3,0.5,1.0,1.0,1.3,11.5,1.5,0.7,0.7,1.0,1.0$, 
$1.1,10.3,22.02 .0,2.0,2.2, .5,1.5,2.5,2.7,4.0,4.0,4.5,3.0,3.0,3.3,3.3,4.7,7.0,7.5,5.0,5.4$, $5.4,8.8,9.0,0.5,0.6,0.6,0.8,0.8,0.7$ and 24.5. Many other useful real data sets are available in Brito et al. (2017), Alizadeh et al. (2018), Korkmaz et al. (2018 and 2019), Cordeiro et al. (2019), Abouelmagd et al. (2019a,b,c), Goual et al. (2019), Goual and Yousof (2019) Yadav etl al. (2019) and Al-Babtain et al. (2020a and b).

The total time test (TTT) plot is an important graphical approach to verify whether the data set can be applied to a specific model or not. Due to Aarset (1987), the empirical version of the TTT plot is given by plotting

$$
T\left(r n^{-1}\right)=\left(\sum_{j=1}^{n} y_{j: n}\right)^{-1} \sum_{j=1}^{r} y_{j: n}+(n-r) y_{j: n},
$$

against $r n^{-1}$, where $r=1, \ldots, n$ and $\left.y_{j: n}\right|_{(j=1, \ldots, n)}$ are the order statistics of the sample. Aarset (1987) showed that the HRF is constant if the TTT plot is graphically presented as a straight diagonal. The HRF is increasing (or decreasing) if the TTT plot is concave (or convex). The HRF is U-shaped (bathtub) if the TTT plot is firstly convex and then concave, if not, the HRF is unimodal. The TTT plots the three real data sets is presented in Figure 2. Plots in Figure 2 indicates that the empirical HRFs of the two data sets are "upside down then bathtub" and upside down respectively. We compare the proposed ZTPTL-Fr distribution with other related
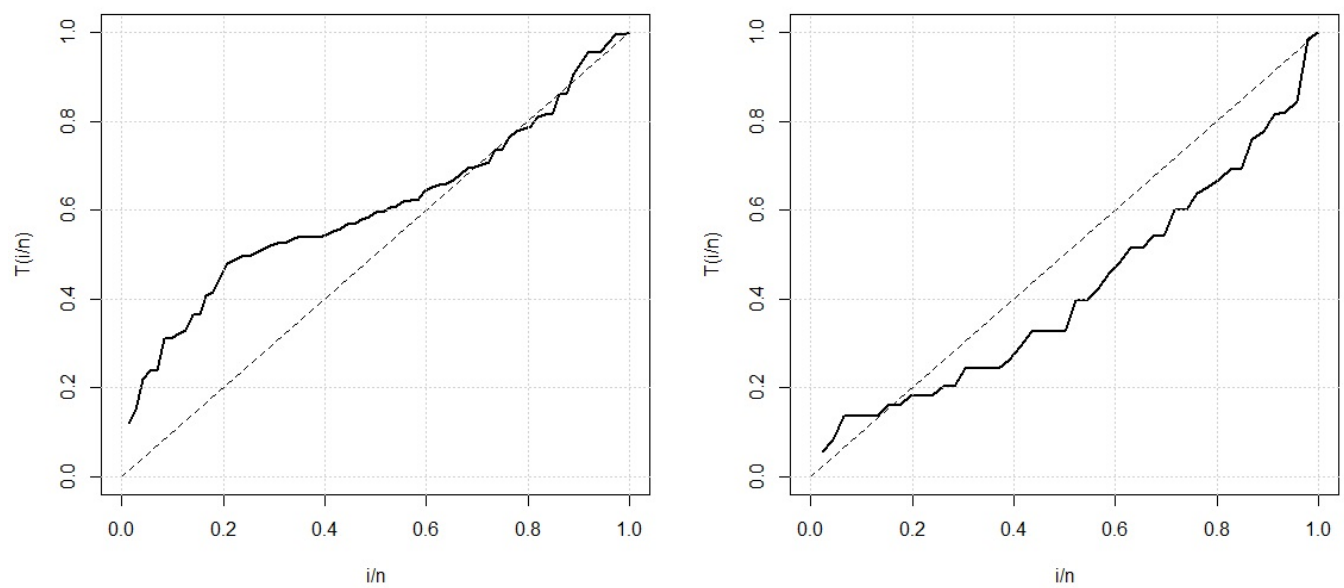

Figure 2: TTT plots for the data set I (left) and data set II (right).

models namely: the Topp Leone Generated Fr (TLG-Fr) , Fr, Kw-Fr, E-Fr, B-Fr, transmuted Fr (T-Fr), MO-Fr and Mcdonald Fr (Mc-Fr) distributions.

Tables 4 and 6 list the values of AIC and BIC however the MLEs and their corresponding standard errors (in parentheses) of the model parameters are listed in Tables 5 and 7 respectively. 


\begin{tabular}{ccc}
\hline \multicolumn{3}{c}{ Table 4: AIC and BIC for data $\mathbf{I}}$. \\
\hline \hline Model & AIC & BIC \\
\hline \hline ZTPTL-Fr & $\mathbf{6 9 6 . 9}$ & $\mathbf{7 0 6 . 1}$ \\
KwMO-Fr & 751.6 & 762.9 \\
E-Fr & 786.5 & 793.3 \\
Kw-Fr & 788.5 & 797.6 \\
ZB-Fr & 787.2 & 794.1 \\
B-Fr & 788.6 & 797.7 \\
KwMOIE & 790.7 & 799.8 \\
MOKw-Fr & 794.2 & 805.6 \\
Fr & 795.3 & 799.9 \\
MO-Fr & 796.1 & 802.9 \\
KwMOIR & 808.2 & 817.3 \\
\hline \hline
\end{tabular}

All values are obtained using the $\mathrm{R}$ program. Figure 3 give the fitted PDF, CDF, HRF, P-P plot and Kaplan-Meier survival plot for data I. Figure 4 give the fitted PDF, CDF, HRF, P-P plot and Kaplan-Meier survival plot for data II. 
Table 5: MLEs and their standard errors (in parentheses) for the survival times for Guinea pigs.

\begin{tabular}{|c|c|c|c|c|c|}
\hline Model & \multicolumn{5}{|c|}{ Estimates } \\
\hline ZTPTL-Fr $(\alpha, \theta, \beta, \delta)$ & & $\begin{array}{c}-3.796 \\
(1.1799)\end{array}$ & $\begin{array}{c}0.0437 \\
(0.000)\end{array}$ & $\begin{array}{c}1.654 \\
(0.098)\end{array}$ & $\begin{array}{c}206.008 \\
(14.38)\end{array}$ \\
\hline $\mathrm{KwMO}-\operatorname{Fr}(a, b, \alpha, \beta, \delta)$ & $\begin{array}{c}0.068 \\
(0.984)\end{array}$ & $\begin{array}{l}54.638 \\
(0.063)\end{array}$ & $\begin{array}{c}308.470 \\
(0.229)\end{array}$ & $\begin{array}{c}0.087 \\
(0.038)\end{array}$ & $\begin{array}{c}69.693 \\
(0.082)\end{array}$ \\
\hline $\mathrm{E}-\operatorname{Fr}(b, \beta, \delta)$ & & & $\begin{array}{l}8.2723 \\
(7.953)\end{array}$ & $\begin{array}{l}0.6207 \\
(0.208)\end{array}$ & $\begin{array}{l}336.3679 \\
(374.803)\end{array}$ \\
\hline $\mathrm{Kw}-\mathrm{Fr}(a, b, \beta, \delta)$ & & $\begin{array}{c}45.7326 \\
(0.092)\end{array}$ & $\begin{array}{l}8.2723 \\
(0.979)\end{array}$ & $\begin{array}{l}0.6207 \\
(0.003)\end{array}$ & $\begin{array}{c}0.7111 \\
(0.013)\end{array}$ \\
\hline $\mathrm{ZB}-\mathrm{Fr}(a, \beta, \delta)$ & & $\begin{array}{l}26.048 \\
(0.597)\end{array}$ & & $\begin{array}{c}1.537 \\
(0.008)\end{array}$ & $\begin{array}{c}6.638 \\
(0.007)\end{array}$ \\
\hline $\mathrm{B}-\operatorname{Fr}(a, b, \beta, \delta)$ & & $\begin{array}{c}19.9786 \\
(7.246)\end{array}$ & $\begin{array}{c}20.1331 \\
(7.26)\end{array}$ & $\begin{array}{c}0.322 \\
(0.00115)\end{array}$ & $\begin{array}{c}24.5032 \\
(0.087)\end{array}$ \\
\hline $\operatorname{KwMOIE}(a, b, \alpha, \delta)$ & $\begin{array}{l}8.8727 \\
(1.174)\end{array}$ & $\begin{array}{c}68.1393 \\
(0.020)\end{array}$ & $\begin{array}{l}2.6258 \\
(0.512)\end{array}$ & & $\begin{array}{l}0.1758 \\
(0.000)\end{array}$ \\
\hline $\mathrm{MOKw}-\operatorname{Fr}(\alpha, a, b, \beta, \delta)$ & $\begin{array}{c}0.449 \\
(0.021)\end{array}$ & $\begin{array}{l}22.880 \\
(3.338)\end{array}$ & $\begin{array}{c}1.376 \\
(0.087)\end{array}$ & $\begin{array}{c}2.666 \\
(0.869)\end{array}$ & $\begin{array}{c}0.449 \\
(0.021)\end{array}$ \\
\hline $\operatorname{Fr}(\beta, \delta)$ & & & & $\begin{array}{c}1.4148 \\
(0.00271)\end{array}$ & $\begin{array}{c}54.1888 \\
(0.111)\end{array}$ \\
\hline $\mathrm{MO}-\operatorname{Fr}(\alpha, \beta, \delta)$ & $\begin{array}{l}14.9816 \\
(4.6305)\end{array}$ & & & $\begin{array}{l}1.7855 \\
(0.193)\end{array}$ & $\begin{array}{l}13.991 \\
(2.964)\end{array}$ \\
\hline $\mathrm{Kw}-\operatorname{MOIR}(a, b, \alpha, \delta)$ & $\begin{array}{c}9.993 \\
(1.972)\end{array}$ & $\begin{array}{c}58.4697 \\
(0.105)\end{array}$ & $\begin{array}{c}0.6389 \\
(0.098)\end{array}$ & & $\begin{array}{l}1.6788 \\
(0.001)\end{array}$ \\
\hline
\end{tabular}

Based on the figures in Tables 4 and 6, we conclude that the ZTPTL-Fr model provide adequate fits as compared to other Fr models in both applications with small values for AIC and BIC. In Application 1, the proposed ZTPTL-Fr model is much better than the B-Fr, E-Fr, MOKw-Fr, MOIE, KwMO-Fr, MO-Fr, Kw-Fr, MOIR and Fr models, so the ZTPTL-Fr model is a good alternative to these models. In Application 2, the proposed ZTPTL-Fr lifetime model is much better than the Fr, T-Fr, Kw-Fr, MO-Fr, TLG-Fr , E-Fr, B-Fr and Mc-Fr models, so the ZTPTL-Fr model a good alternative to these models. 
Table 6: AIC and BIC for data II.

\begin{tabular}{ccc}
\hline \hline Model & AIC & BIC \\
\hline \hline ZTPTL-Fr & $\mathbf{1 4 4 . 3}$ & $\mathbf{1 5 1 . 6}$ \\
TLG-Fr & 207.2 & 214.5 \\
Fr & 207.4 & 215.0 \\
Kw-Fr & 207.4 & 214.6 \\
E-Fr & 207.4 & 214.9 \\
B-Fr & 207.4 & 214.7 \\
T-Fr & 207.8 & 215.3 \\
MO-Fr & 207.9 & 214.7 \\
Mc-Fr & 207.8 & 216.9 \\
\hline \hline
\end{tabular}

\section{Conclusions}

In this paper, a new four parameter zero truncated Poisson Fr distribution called the zerotruncated Poisson Topp Leone Fr (ZTPTL-Fr) model is defined and studied. Various structural mathematical properties of the proposed extreme value model including ordinary and incomplete moments, residual and reversed residual life functions generating functions and order statistics are investigated. The maximum likelihood method is used to estimate the model parameters. The new distribution is applied for modeling two real data sets to illustrate empirically its flexibility. The ZTPTL-Fr model provide adequate fits as compared to other Fr models in both applications with small values for AIC and BIC. The proposed ZTPTL-Fr model is much better than Marshall-Olkin Kumaraswamy Fr, beta Fr, Marshall-Olkin Fr, Kumaraswamy Fr, the Kumaraswamy-Marshall-Olkin -Fr, Marshall-Olkin inverse exponential, Marshall-Olkin inverse Rayleigh, exponentiated Fr and Fr models, so the ZTPTL-Fr model is a good alternative to these models for modeling survival times data. As well as the proposed ZTPTL-Fr lifetime model is much better than the Fr, Transmuted Fr, Kumaraswamy Fr, Topp Leone Generated Fr, exponentiated Fr, beta Fr, Marshall-Olkin Fr and Mcdonald Fr models, so the ZTPTL-Fr model a good alternative to these models for modeling repair times data. We assess the performance of the maximum likelihood method by means of a numerical simulation study, We observe that our estimates are pretty stable especially when $n \geq 300$ and as $n$ increases the MSEs decreases. So, the maximum likelihood method works very well to estimate the model parameters. 
Table 7: MLEs and their standard errors (in parentheses) for the repair times data.

\begin{tabular}{|c|c|c|c|c|c|}
\hline Model & \multicolumn{5}{|c|}{ Estimates } \\
\hline ZTPTL-Fr $(\alpha, \theta, \beta, \delta)$ & $\begin{array}{c}-7.3631 \\
1.566 \times \mathrm{e}^{-1}\end{array}$ & $\begin{array}{c}0.0026 \\
5.586 \times \mathrm{e}^{-4}\end{array}$ & & $\begin{array}{c}1.0887 \\
3.163 \times \mathrm{e}^{-1}\end{array}$ & $\begin{array}{c}48.2580 \\
5.8795 \times \mathrm{e}^{1}\end{array}$ \\
\hline TLG-Fr $(a, b, \beta, \delta)$ & $\begin{array}{c}0.1405 \\
(0.2299)\end{array}$ & $\begin{array}{c}2.1672 \\
(20.072)\end{array}$ & & $\begin{array}{c}0.8958 \\
(0.1675)\end{array}$ & $\begin{array}{c}4.9552 \\
(51.257)\end{array}$ \\
\hline $\operatorname{Fr}(\beta, \delta)$ & & & & $\begin{array}{c}1.0128 \\
(0.1129)\end{array}$ & $\begin{array}{c}1.1297 \\
(0.1740)\end{array}$ \\
\hline $\mathrm{Kw}-\operatorname{Fr}(a, b, \beta, \delta)$ & $\begin{array}{l}1.1619 \\
(7.452)\end{array}$ & $\begin{array}{l}3.8034 \\
(4.604)\end{array}$ & & $\begin{array}{c}0.5401 \\
(0.2753)\end{array}$ & $\begin{array}{c}4.0226 \\
(47.459)\end{array}$ \\
\hline $\mathrm{E}-\operatorname{Fr}(a, \beta, \delta)$ & $\begin{array}{c}0.9881 \\
(23.679)\end{array}$ & & & $\begin{array}{c}1.0125 \\
(0.1129)\end{array}$ & $\begin{array}{c}1.1433 \\
(27.057)\end{array}$ \\
\hline $\mathrm{B}-\operatorname{Fr}(a, b, \beta, \delta)$ & $\begin{array}{l}2.3521 \\
(8.581)\end{array}$ & $\begin{array}{c}5.8362 \\
(14.877)\end{array}$ & & $\begin{array}{c}0.4147 \\
(0.5619)\end{array}$ & $\begin{array}{c}3.4905 \\
(13.461)\end{array}$ \\
\hline $\mathrm{T}-\operatorname{Fr}(a, \beta, \delta)$ & $\begin{array}{l}-0.6364 \\
(0.1173)\end{array}$ & & & $\begin{array}{c}1.0853 \\
(0.1226)\end{array}$ & $\begin{array}{c}0.7747 \\
(0.3633)\end{array}$ \\
\hline $\operatorname{MO}-\operatorname{Fr}(a, \beta, \delta)$ & $\begin{array}{c}4.9168 \\
(6.1834)\end{array}$ & & & $\begin{array}{c}1.3384 \\
(0.2574)\end{array}$ & $\begin{array}{c}0.5066 \\
(0.3068)\end{array}$ \\
\hline $\operatorname{Mc}-\operatorname{Fr}(a, b, \alpha, \beta, \delta)$ & $\begin{array}{c}0.0125 \\
(0.0108)\end{array}$ & $\begin{array}{c}96.427 \\
(354.85)\end{array}$ & $\begin{array}{c}0.8957 \\
(0.1297)\end{array}$ & $\begin{array}{c}12.281 \\
(45.502)\end{array}$ & $\begin{array}{c}10.570 \\
(35.124)\end{array}$ \\
\hline
\end{tabular}




\section{References}

[1] Abouelmagd, T. H. M., Hamed, M. S., Almamy, J. A., Ali, M. M., Yousof, H. M. and Korkmaz, M. C. (2019a). Extended Weibull log-logistic distribution. Journal of Nonlinear Sciences and Applications, 12(8), 523-534.

[2] Abouelmagd, T. H. M., Hamed, M. S., Hamedani G. G. , Ali, M. M., Goual, H., Korkmaz, M. C. and Yousof, H. M. (2019b). The zero truncated Poisson Burr X family of distributions with properties, characterizations, applications, and validation test. Journal of Nonlinear Sciences and Applications, 12(5), 314-336.

[3] Abouelmagd, T. H. M., Hamed. M. S. Handique, L. Goual, H. Ali, M. M., Yousof, H. M. and Korkmaz, M. C. (2019c). A new class of distributions based on the zero truncated Poisson distribution with properties and applications. Journal of Nonlinear Sciences and Applications, 12(3), 152-164.

[4] Al-Babtain, A. A. Elbatal, I. and Yousof, H. M. (2020a). A new flexible three-parameter model: properties, Clayton Copula, and modeling real data, Symmetry, 12, 1-17. doi:10.3390/sym12030440

[5] Al-Babtain, A. A. Elbatal, I. and Yousof, H. M. (2020b). A new three parameter Fréchet model with mathematical properties and applications. Journal of Taibah University for Science, 14(1), 265-278.

[6] Afify, A. Z., Hamedani, G. G. Ghosh, I. and Mead, M. E. (2015). The transmuted MarshallOlkin Fréchet distribution: properties and applications. International Journal Statistics Probability, 4, 132-184.

[7] Afify, A. Z., Yousof, H. M., Cordeiro, G.M. and Ahmad, M. (2016a). The Kumaraswamy Marshall-Olkin Fréchet distribution with applications. Journal of Islamic Countries Society of Statistical Sciences, 2, 1-18.

[8] Afify, A. Z., Yousof, H. M., Cordeiro, G. M., Ortega, E. M. M. and Nofal, Z. M. (2016b). The Weibull Fréchet distribution and its applications. Journal of Applied Statistics, 43, 2608-2626.

[9] Afify, A. Z., Yousof, H. M. and Nadarajah, S. (2016c). The beta transmuted-H family of distributions: properties and applications. Stasistics and its Inference, 10, 505-520.

[10] Alizadeh, M., Rasekhi, M., Yousof, H. M. and Hamedani G. G. (2018). The transmuted Weibull G family of distributions. Hacettepe Journal of Mathematics and Statistics, 47(6), $1-20$.

[11] Barreto-Souza, W. M., Cordeiro, G. M. and Simas, A. B. (2011). Some results for beta Fréchet distribution. Commun. Statist. Theory-Meth., 40, 798-811.

[12] Brito, E., Cordeiro, G. M., Yousof, H. M., Alizadeh, M. and Silva, G. O. (2017). ToppLeone Odd Log-Logistic Family of Distributions, Journal of Statistical Computation and Simulation, 87(15), 3040-3058. 
[13] Cordeiro, G. M., Yousof, H. M., Ramires, T. G. and Ortega, E. M. M. (2018). The Burr XII system of densities: properties, regression model and applications. Journal of Statistical Computation and Simulation, 88(3), 432-456.

[14] Elbatal, I. Asha, G. and Raja, V. (2014). transmuted exponentiated Fréchet distribution: properties and applications. Journal of Statistics Applications and Probability, 3, 379-394.

[15] Fisher, R. A. and Tippett, L. H. C. (1928). Limiting forms of the frequency distribution of the largest or smallest member of a sample. In Mathematical Proceedings of the Cambridge Philosophical Society, pages 180-190. Cambridge Univ Press.

[16] Fréchet, M. (1927). Sur la loi de probabilité de lécart maximum. Ann. de la Soc. polonaisede Math, 6, 93-116.

[17] Gnedenko, B. (1943). Sur la distribution limite du terme maximum d'une serie aleatoire. Annals of Mathematics, Volume 44.

[18] Goual, H. and Yousof, H. M. (2019). Validation of Burr XII inverse Rayleigh model via a modified chi-squared goodness-of-fit test. Journal of Applied Statistics, 47(1), 1-32.

[19] Goual, H., Yousof, H. M. and Ali, M. M. (2019). Validation of the odd Lindley exponentiated exponential by a modified goodness of fit test with applications to censored and complete data. Pak. J. Stat. Oper. Res. 15(3), 745-771.

[20] Harlow, D. G. (2002). Applications of the Fréchet distribution function, Int. J. Mater. Prod. Technol, 17, 482-495.

[21] Korkmaz, M. C., Alizadeh, M., Yousof, H. M. and Butt, N. S. (2018). The generalized odd Weibull generated family of distributions: statistical properties and applications. Pak. J. Stat. Oper. Res., 14(3), 541-556.

[22] Korkmaz, M. C., Altun, E., Yousof, H. M. and Hamedani G. G. (2019). The odd power Lindley generator of probability distributions: properties, characterizations and regression modeling, International Journal of Statistics and Probability, 8(2), 70-89.

[23] Korkmaz, M. C. Yousof, H. M. and Ali, M. M. (2017). Some theoretical and computational aspects of the odd Lindley Fréchet distribution, Journal of Statisticians: Statistics and Actuarial Sciences, 2, 129-140.

[24] Kotz, S. and Johnson, N. L. (1992). Breakthroughs in Statistics: Foundations and basic theory. Springer, Volume 1.

[25] Kotz, S. and Nadarajah, S. (2000). Extreme Value Distributions: Theory and Applications, Imperial College Press, London.

[26] Krishna, E., Jose, K. K., Alice, T. and Risti , M. M. (2013). The Marshall-Olkin Fréchet distribution. Communications in Statistics-Theory and Methods, 42, 4091-4107.

[27] Mahmoud, M. R. and Mandouh, R. M. (2013). On the transmuted Fréchet distribution. Journal of Applied Sciences Research, 9, 5553-5561. 
[28] Nadarajah, S. and Gupta, A. K. (2004). The beta Fréchet distribution. Far East Journal of Theoretical Statistics, 14, 15-24.

[29] Nadarajah, S. and Kotz, S. (2003). Moments of some J-shaped distributions. Journal of Applied Statistics, 30, 311-317.

[30] Nadarajah, S. and Kotz, S. (2008). Sociological models based on Fréchet random variables. Quality and Quantit, 42, 89-95.

[31] Sindhu, T. N., Saleem, M. and Aslam, M. (2013). Bayesian estimation for Topp Leone distribution under trimmed samples. Journal of Basic and Applied Scientific Research, 3, 347-360.

[32] Von Mises, R. (1936). La distribution de la plus grande de n valeurs. Rev. math. Union interbalcanique, Volume 1.

[33] Von Mises, R. (1964). Selected papers of Richard von Mises, American mathematical society, Volume 1.

[34] Yadav, A.S., Goual, H., Alotaibi, R.M. Rezk, H., Ali, M.M. and Yousof, H.M. (2020). Validation of the Topp-Leone-Lomax model via a modified Nikulin-Rao-Robson goodness-of-fit test with different methods of estimation. Symmetry, 12, 1-26. doi: 10.3390/sym12010057

[35] Yousof, H. M., Afify, A. Z., Alizadeh, M., Butt, N. S., Hamedani, G. G. and Ali, M. M. (2015). The transmuted exponentiated generalized-G family of distributions. Pakistan Journal of Statistics and Operation Research , 11, 441-464.

[36] Yousof, H. M., Afify, A. Z., Ebraheim, A. N., Hamedani, G. G. and Butt, N. S. (2016). On six-parameter Fréchet distribution: properties and applications. Pakistan Journal of Statistics and Operation Research, 12, 281-299.

[37] Yousof, H. M., Alizadeh, M., Jahanshahi, S. M. A., Ramires, T. G., Ghosh, I. and Hamedani G. G. (2017). The transmuted Topp-Leone G family of distributions: theory, characterizations and applications. Journal of Data Science, 15, 723-740.

[38] Yousof, H. M., Altun, E. and Hamedani G. G. (2018a). A new extension of Fréchet distribution with regression models, residual analysis and characterizations. Journal of Data Science, 16, 743-770.

[39] Yousof, H. M., Jahanshahi, S. M., Ramires, T. G Aryal, G. R. and Hamedani G. G. (2018b). A new distribution for extreme values: regression model, characterizations and applications. Journal of Data Science, 16, 677-706. 

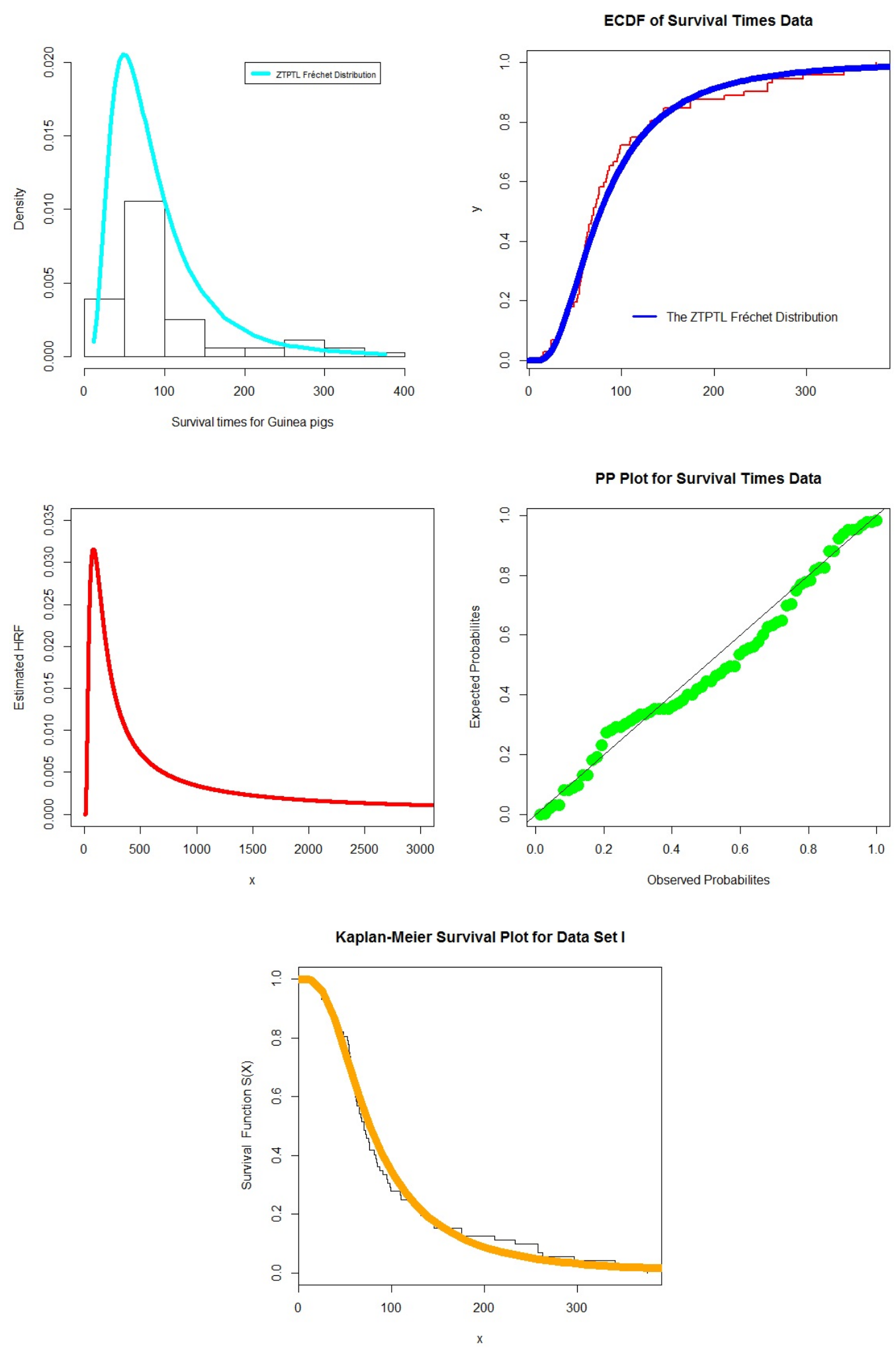

Figure 3: The fitted PDF, CDF, HRF, P-P plot and Kaplan-Meier survival plot for the first data set. 

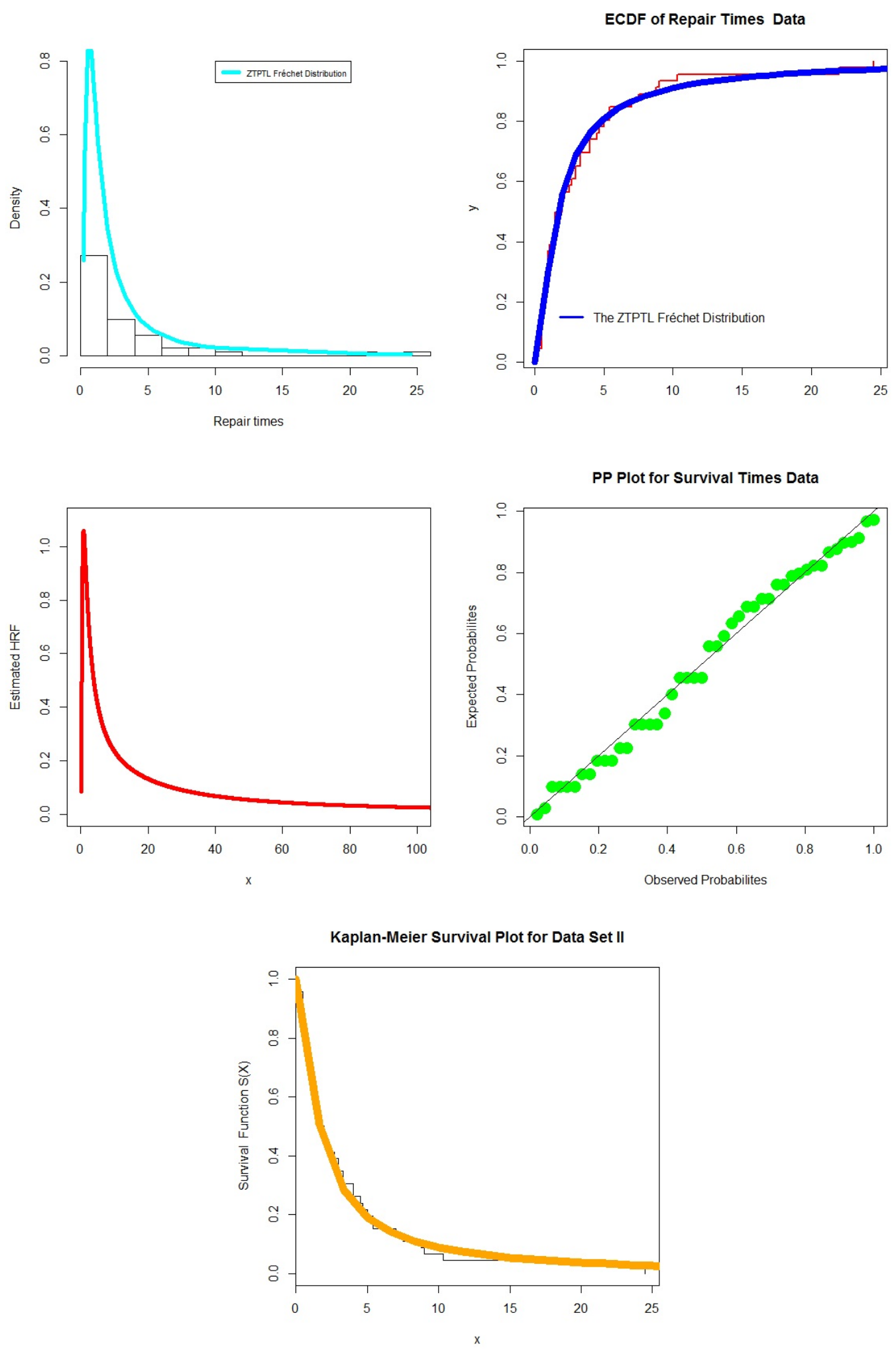

Figure 4: The fitted PDF, CDF, HRF, P-P plot and Kaplan-Meier survival plot for the second data set. 